\title{
Timoshenko's Shear Coefficient for Transverse Vibrations of Trigonal Crystals of Rectangular Cross Section*
}

\author{
Hirofumi KAWASHIMA**
}

\begin{abstract}
In this paper, we derive the shear coefficient $K$ for trigonal crystals of rectangular cross section in Timoshenko's beam theory of three dimensional (3-D) elasticity. The theoretical result gives $K$ as a function of the transformed elastic stiffness constants $c_{22}^{\prime}, \hat{c}_{44}, c_{66}^{\prime}$. For an isotropic material it agrees completely with that derived by G. R. Cowper. In addition, values of $K$ versus cut angle for quartz crystal, lithium tantalate, and lithium niobate which belong to the trigonal system are calculated, so that $K$ has a value of $0.796-0.867$ for quartz crystal, $0.834-0.857$ for lithium tantalate, and 0.831 0.863 for lithium niobate, when $Z$-plates of the crystals are rotated with a cut angle $\theta$ of $0-180^{\circ}$ about the $x$-axis. The values of $K$ are then compared with those for isotropic materials obtained by other authors.
\end{abstract}

Key Words: Piezoelement, Timoshenko's Shear Coefficient, Trigonal Crystal, Rectangular Cross Section, Transverse Vibration

\section{Introduction}

The one-dimensional (1-D) theory of beams can be extended in the range of applicability by taking account of rotary inertia and transverse shear deformations. The equations with these effects are generally referred to as Timoshenko's beam equations ${ }^{(1)}$. In these equations, the effective transverse shear strain is taken as equal to the average shear stress on a cross section divided by the product of the shear modulus (elastic stiffness constant) and the shear coefficient, $K$. In addition, the shear coefficient $K$ is commonly defined as the ratio of the average shear strain on a section to the shear strain at the centroid.

However, there occurs a big difference between the results analyzed using the commonly defined $K$ and the experimental results ${ }^{(2)}$. Leibowitz and Kennard have criticized the definition, pointing out an unwarranted assumption in the underlying analysis,

* Received 18th September, 1996. Japanese original : Trans. Jpn. Soc. Mech. Eng., Vol. 62, No. 598, C (1996), pp. 2095-2101 (Received 9th August, 1995)

** Seiko Instruments Inc., 563 Takatsuka-shinden, Matsudo-shi, Chiba 271, Japan and Cowper has agreed with their criticism and has pointed out that further study of the shear coefficient is needed ${ }^{(2)}$. In addition, Mindlin, et al. ${ }^{(3)}$, and Goodman $^{(4)}$ have pointed out that Timoshenko's beam equations with the customary values of $K$ lead to unsatisfactory results when the high resonance frequencies of vibrating beams are calculated, and have insisted that $K$ should be adjusted arbitrarily in order to obtain better results. However, they do not discuss the basic definition of $K$. Instead, they point out that the distribution of shear strain over a cross section varies with the mode of vibration, i.e., frequency.

According to the thinking of Leibowitz and Kennard, Cowper has examined the shear coefficient $K$ for an isotropic material in detail and better results are obtained $^{(2)}$. It must be noted, however, that $K$ for an anisotropic material such as quartz crystal, lithium tantalate, and lithium niobate is not examined at all.

In this paper, the shear coefficient $K$ for trigonal crystals which are anisotropic is described. In analysis procedure, first, the shear coefficient $K$ in Timoshenko's beam theory is derived using Cowper's definition and is found to be given as a function of stress-component $T_{6}$ and residual displacement $v_{y}$. 
Next, $T_{6}$ and $v_{y}$ are derived from the equations of equilibrium and the boundary conditions. Substitution of $T_{6}$ and $v_{y}$ into $K$ gives a function of harmonic function $\chi^{\prime}$. The shear coefficient $K$ is, therefore, calculated using $\chi^{\prime}$ derived. Finally, the calculated values of $K$ for quartz crystal, lithium tantalate, and lithium niobate are shown to a cut angle rotated about the $x$-axis.

\section{Derivative of shear coefficient $K$}

Figure 1 shows a flexural crystal resonator of a beam which belongs to a trigonal system, and its coordinate system (a), and front view and sectional view when lateral force $Q$ is applied at the end (b). Now, as length $2 y_{0}$ is much larger than width $2 x_{0}$ and thickness $2 z_{0}$, stress-components $T_{1}, T_{3}, T_{5}$ become

$$
T_{1}=T_{3}=T_{5}=0
$$

For the rotation about the $x$-axis (rotation angle $\theta$ ), a relationship between stress $T_{p}$ and strain $S_{q}$ for trigonal crystals is given as

$$
\begin{aligned}
& T_{1}=c_{11} S_{1}+c_{12} S_{2}+c_{13} S_{3}+c_{14} S_{4} \\
& T_{2}=c_{21} S_{1}+c_{22} S_{2}+c_{23} S_{3}+c_{24} S_{4} \\
& T_{3}=c_{31} S_{1}+c_{32} S_{2}+c_{33} S_{3}+c_{34} S_{4} \\
& T_{4}=c_{41} S_{1}+c_{42} S_{2}+c_{43} S_{3}+c_{44} S_{4} \\
& T_{5}=c_{55} S_{5}+c_{56} S_{6} \\
& T_{6}=c_{65} S_{5}+c_{66} S_{6}
\end{aligned}
$$

where $c_{p q}$ is elastic stiffness constant. Accordingly, from the relation of Eq. (1), Eq. (2) is simplified into

$$
\begin{aligned}
& T_{2}=c_{22}^{\prime} S_{2}+c_{24}^{\prime} S_{4} \\
& T_{4}=c_{2}^{\prime} S_{2}+c_{44}^{\prime} S_{4} \\
& T_{6}=c_{66}^{\prime} S_{6}
\end{aligned}
$$

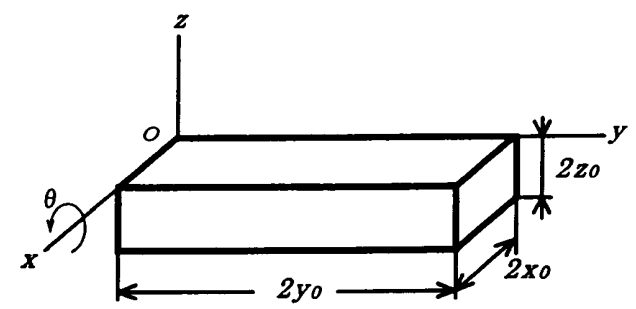

(a)

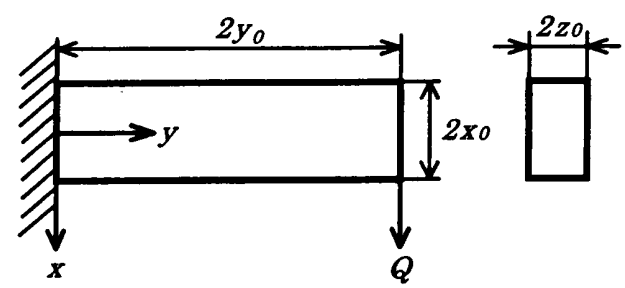

( b )

Fig. 1 Flexural quartz crystal resonator of beam and its coordinate system (a), and front view and sectional view when lateral force $Q$ is applied at the end ( $b$ ) where,

$$
\begin{aligned}
& c_{p q}^{\prime}=\bar{c}_{p q}-\bar{c}_{1 p} \bar{c}_{q 1} / \bar{c}_{11} \quad(p, q=2,4) \\
& \bar{c}_{p q}=c_{p q}-c_{3 p} c_{q 3} / c_{33} \quad(p, q=1,2,4) \\
& c_{66}^{\prime}=c_{66}-c_{56} C_{65} / c_{55}
\end{aligned}
$$

The definition of displacement and rotation angle accords to Ref. (2). The transverse mean deflection $U(y)$ of the cross section and the mean displacement $V(y)$ of the cross section in the $y$-axis direction are defined as

$$
U(y)=\frac{1}{A} \int_{-z_{0}}^{z_{0}} \int_{-x_{0}}^{x_{0}} u_{x} d x d z
$$

and

$$
V(y)=\frac{1}{A} \int_{-z_{0}}^{z_{0}} \int_{-x_{0}}^{x_{0}} u_{y} d x d z
$$

where $A$ is the area of the cross section, $u_{x}$ and $u_{y}$ are, respectively, the $x$-component and the $y$-component of displacement of a point of the beam. In addition, the mean angle $\Phi(y)$ of rotation of a cross section about the neutral axis is also defined as

$$
\Phi(y)=\frac{1}{I_{z}} \int_{-z_{0}}^{z_{0}} \int_{-x_{0}}^{x_{0}} x u_{y} d x d z
$$

where,

$$
I_{z}=\int_{-z_{0}}^{z_{0}} \int_{-x_{0}}^{x_{0}} x^{2} d x d z
$$

Further, residual displacements $v_{x}, v_{y}$ are defined by the following relation, namely

$$
\begin{aligned}
& u_{x}=U(y)+v_{x} \\
& u_{y}=V(y)+x \Phi(y)+v_{y}
\end{aligned}
$$

It follows from Eq. $(7)$ that

$$
\begin{gathered}
\int_{-z_{0}}^{z_{0}} \int_{-x_{0}}^{x_{0}} v_{x} d x d z=\int_{-z_{0}}^{z_{0}} \int_{-x_{0}}^{x_{0}} v_{y} d x d z \\
=\int_{-z_{0}}^{z_{0}} \int_{-x_{0}}^{x_{0}} x v_{y} d x d z=0
\end{gathered}
$$

The residual displacement $v_{y}$ essentially represents the warping of the cross section. From Eq. (3) the stress-strain relation is

$$
T_{6}=c_{66}^{\prime}\left(\frac{\partial u_{x}}{\partial y}+\frac{\partial u_{y}}{\partial x}\right)
$$

Substitution of Eq. ( 7 ) into Eq. ( 9 ) gives

$$
\frac{\partial U(y)}{\partial y}+\Phi(y)=\frac{T_{6}}{c_{66}^{\prime}}-\frac{\partial v_{x}}{\partial y}-\frac{\partial v_{y}}{\partial x}
$$

Integration of Eq. (10) over the cross section then yields, in view of Eq. ( 8$)$,

$$
\frac{\partial U(y)}{\partial y}+\Phi(y)=\frac{1}{A c_{66}^{\prime}} \int_{-z_{0}}^{z_{0}} \int_{-x_{0}}^{x_{0}}\left(T_{6}-c_{66}^{\prime} \frac{\partial v_{y}}{\partial x}\right) d x d z
$$

The right term of Eq. (11) can be replaced by

$$
\frac{Q}{K A c_{66}^{\prime}}
$$

The $K$ of Eq. (12) is called Timoshenko's shear coefficient. From the relation of Eqs. (11) and (12) the shear coefficient $K$ is rewritten as

$$
K=\frac{Q}{\int_{-z_{0}}^{z_{0}} \int_{-x_{0}}^{x_{0}}\left(T_{6}-c_{66}^{\prime} \frac{\partial v_{y}}{\partial x}\right) d x d z}
$$

That is, the shear coefficient per unit force $K / Q$ is 
given as a function of stress $T_{6}$, elastic stiffness constant $c_{66}^{\prime}$ and residual displacement $v_{y}$. The stress $T_{6}$ and the residual displacement $v_{y}$ are derived next.

\section{Derivative of stress $T_{6}$ and residual displacement $v_{y}$}

\section{1 Equation of equilibrium}

The bending moment $M$ at the cross section distant $y$ from the fixed end is

$$
M=Q\left(2 y_{0}-y\right)
$$

Here, we assume that stress-component $T_{2}$ on any element of this section is given by the equation

$$
T_{2}=-Q\left(2 y_{0}-y\right) x / I_{z}
$$

Since two of the equations of equilibrium from the relation of Eq. (1) must be satisfied with

$$
\frac{\partial T_{6}}{\partial y}=0 \text { and } \frac{\partial T_{4}}{\partial y}=0
$$

it follows that $T_{6}$ and $T_{4}$ must be independent of $y$. By use of the relation of Eq. (15), the equation of equilibrium reduces to

$$
\frac{\partial T_{6}}{\partial x}+\frac{\partial T_{4}}{\partial z}+\frac{Q}{I_{z}} x=0
$$

In addition, there is a relationship with respect to strain $S_{q}(q=3$ to 6$)$ as follows :

$$
\frac{\partial}{\partial z}\left(\frac{\partial S_{4}}{\partial x}+\frac{\partial S_{5}}{\partial y}-\frac{\partial S_{6}}{\partial z}\right)=2 \frac{\partial^{2} S_{3}}{\partial x \partial y}
$$

From the relation of Eqs. ( 2 ) and (16), it follows that $\partial S_{5} / \partial y=0 . \quad$ Equation (18) is, therefore transformed into

$$
\frac{\partial S_{4}}{\partial x}-\frac{\partial S_{6}}{\partial z}=2 \tau-\frac{2 \sigma_{z}}{C_{22}^{\prime}} \frac{Q}{I_{z}} z
$$

where $2 \tau$ is a constant of integration and $\sigma_{z}$ is a dimensionless constant which is given by elastic stiffness constants; and from this equation it follows that $S_{4}$ and $S_{6}$ which satisfy Eq. (19) can be expressed in the forms

$$
\begin{aligned}
& S_{4}=\tau x+\frac{\partial \phi_{0}}{\partial z} \\
& S_{6}=-\tau z+\frac{\partial \phi_{0}}{\partial x}+\frac{\sigma_{z}}{c_{22}^{\prime}} \frac{Q}{I_{z}} z^{2}
\end{aligned}
$$

where $\phi_{0}$ is a function of $x$ and $z$.

From Eqs. (20) and ( 3 ) the equation of equilibrium of Eq. (17) reduces to

$$
c_{66}^{\prime} \frac{\partial^{2} \phi_{0}}{\partial x^{2}}+\widehat{c}_{44} \frac{\partial^{2} \phi_{0}}{\partial z^{2}}+\frac{Q}{I_{z}} x=0
$$

where, $\hat{c}_{44}=c_{44}^{\prime}-c_{24}^{\prime 2} / c_{22}^{\prime}\left(\because c_{24}^{\prime}=c_{42}^{\prime}\right)$.

In addition, we change the variables by putting

$$
\begin{aligned}
& x^{\prime}=x \sqrt{\frac{\hat{c}_{44}+c_{66}^{\prime}}{2 c_{66}^{\prime}}}, z^{\prime}=z \sqrt{\frac{\widehat{c}_{44}+c_{66}^{\prime}}{2 \widehat{c}_{44}}}, \\
& \phi_{0}^{\prime}=\phi_{0} \frac{\widehat{c}_{44}+c_{66}^{\prime}}{2 \sqrt{\hat{C}_{44} c_{66}^{\prime}}}
\end{aligned}
$$

The equation of equilibrium of Eq. (21) is then rewritten as

$$
\frac{\partial^{2} \phi_{0}^{\prime}}{\partial x^{\prime 2}}+\frac{\partial^{2} \phi_{0}^{\prime}}{\partial z^{\prime 2}}+\frac{Q}{c_{22}^{\prime} I_{z}} \Xi x^{\prime}=0
$$

where,

$$
\Xi=\frac{c_{22}^{\prime}}{\widehat{c}_{44}} \sqrt{\frac{2 \bar{c}_{44}}{\hat{c}_{44}+c_{66}^{\prime}}}
$$

\subsection{Boundary condition}

On the other hand, the condition that the bounding surface is free from traction must be

$$
T_{6} \cos (x, \nu)+T_{4} \cos (z, \nu)=0
$$

Here $\nu$ denotes the direction of the normal to a plane, and the general form of $\cos (a, \nu)$ has the following relation

$$
\cos (a, \nu)= \begin{cases}1 & |\nu|=a \\ 0 & |\nu| \neq a\end{cases}
$$

From the relations of Eqs. (3),(15) and (20), the equation of the boundary condition becomes

$$
\begin{aligned}
& \frac{\partial \phi_{0}}{\partial \nu}=\tau\{z \cos (x, \nu)-x \cos (z, \nu)\} \\
& \quad-\frac{\sigma_{z}}{c_{22}^{\prime}} \frac{Q}{I_{z}} z^{2} \cos (x, \nu) \\
& \quad+\frac{Q}{C_{22}^{\prime} I_{z}} \frac{c_{42}^{\prime}}{\hat{C}_{44}}\left(2 y_{0}-y\right) x \cos (z, \nu)
\end{aligned}
$$

which holds at all points of the bounding curve of any cross section. Here $d \nu$ denotes the element of the outward-drawn normal to this curve. In addition, if $f(x, z)=0$ is the equation of the bounding curve, cos $(x, \nu)$ and $\cos (z, \nu)$ become equal to $\partial f / \partial x$ and $\partial f / \partial z$, respectively. Now, when a new function $F\left(x^{\prime}, z^{\prime}\right)$ is satisfied with

$$
F\left(x^{\prime}, z^{\prime}\right) \equiv f\left(x^{\prime} \sqrt{\frac{2 c_{66}^{\prime}}{\widehat{c}_{44}+c_{66}^{\prime}}}, z^{\prime} \sqrt{\frac{2 \widehat{c}_{44}}{\hat{c}_{44}+c_{66}^{\prime}}}\right)
$$

the equation $f(x, z)=0$ becomes $F\left(x^{\prime}, z^{\prime}\right)=0$. Therefore, by taking account of Eq. (27) and the relation of $\partial F / \partial x^{\prime}=\cos \left(x^{\prime}, \nu^{\prime}\right)$ and $\partial F / \partial z^{\prime}=\cos \left(z^{\prime}, \nu^{\prime}\right)$, the equation of the boundary condition of Eq. (26) can be rewritten as

$$
\begin{aligned}
& \frac{\partial \phi_{0}^{\prime}}{\partial \nu^{\prime}}=\tau\left\{z^{\prime} \cos \left(x^{\prime}, \nu^{\prime}\right)-x^{\prime} \cos \left(z^{\prime}, \nu^{\prime}\right)\right\} \\
& \quad-\frac{Q}{C_{22}^{\prime} I_{z}} \Xi^{\prime} \sigma_{z} z^{\prime 2} \cos \left(x^{\prime}, \nu^{\prime}\right) \\
& \quad+\frac{Q}{C_{22}^{\prime} I_{z}} \frac{C_{42}^{\prime}}{\hat{C}_{44}}\left(2 y_{0}-y\right) x^{\prime} \cos \left(z^{\prime}, \nu^{\prime}\right)
\end{aligned}
$$

where,

$$
\Xi^{\prime}=\sqrt{\frac{2 \hat{c}_{44}}{\hat{c}_{44}+c_{66}^{\prime}}}
$$

Here $d \nu^{\prime}$ is the element of the normal to the transformed boundary. Equations (23) and (28) are further simplified by putting

$$
\begin{aligned}
\phi_{0}^{\prime} & =\tau \phi^{\prime}-\frac{Q}{c_{22}^{\prime} I_{z}}\left\{\chi^{\prime}-\frac{1}{6}\left(1-\frac{1}{2} \Xi\right) x^{\prime 3}\right. \\
& \left.+\frac{1}{2}\left(1+\frac{1}{2} \Xi\right) x^{\prime} z^{\prime 2}\right\}
\end{aligned}
$$

then $\phi^{\prime}$ is the torsion function for the cross section. Substitution of Eq. (29) into Eq. (23) gives $\chi^{\prime}$ and $\phi^{\prime}$ which are, respectively, satisfied with

$\frac{\partial^{2} \chi^{\prime}}{\partial x^{\prime 2}}+\frac{\partial^{2} \chi^{\prime}}{\partial z^{\prime 2}}=0$ 
and

$$
\frac{\partial^{2} \phi^{\prime}}{\partial x^{\prime 2}}+\frac{\partial^{2} \phi^{\prime}}{\partial z^{\prime 2}}=0
$$

From Eq. (29) $\partial \phi_{0}^{\prime} / \partial \nu^{\prime}$ becomes

$$
\begin{aligned}
& \frac{\partial \phi_{0}^{\prime}}{\partial \nu^{\prime}}=\tau\left\{\frac{\partial \phi^{\prime}}{\partial x^{\prime}} \cos \left(x^{\prime}, \nu^{\prime}\right)+\frac{\partial \phi^{\prime}}{\partial z^{\prime}} \cos \left(z^{\prime}, \nu^{\prime}\right)\right\} \\
& \quad-\frac{Q}{c_{22}^{\prime} I_{z}}\left[\left\{\frac{\partial \chi^{\prime}}{\partial x^{\prime}}-\frac{1}{2}\left(1-\frac{1}{2} \Xi\right) x^{\prime 2}\right.\right. \\
& \left.\quad+\frac{1}{2}\left(1+\frac{1}{2} \Xi\right) z^{\prime 2}\right\} \cos \left(x^{\prime}, \nu^{\prime}\right) \\
& \left.\quad+\left\{\frac{\partial \chi^{\prime}}{\partial z^{\prime}}+\left(1+\frac{1}{2} \Xi\right) x^{\prime} z^{\prime}\right\} \cos \left(z^{\prime}, \nu^{\prime}\right)\right]
\end{aligned}
$$

Substituting this relation into Eq. (28), $\partial \chi^{\prime} / \partial \nu^{\prime}$ yields

$$
\begin{aligned}
\frac{\partial \chi^{\prime}}{\partial \nu^{\prime}} & =\left\{\frac{1}{2}\left(1-\frac{1}{2} \Xi\right) x^{\prime 2}\right. \\
& \left.-\frac{1}{2}\left(1+\frac{1}{2} \Xi-2 \Xi^{\prime} \sigma_{z}\right) z^{\prime 2}\right\} \cos \left(x^{\prime}, \nu^{\prime}\right) \\
& -\left\{\left(1+\frac{1}{2} \Xi\right) z^{\prime}+\frac{C_{42}^{\prime}}{\widehat{C}_{44}}\left(2 y_{0}-y\right)\right\} x^{\prime} \cos \left(z^{\prime}, \nu^{\prime}\right)
\end{aligned}
$$

\section{3 Stress $T_{6}$}

Since strains $S_{4}$ and $S_{6}$ are, respectively, given as a function of $\partial \phi_{0} / \partial x$ and $\partial \phi_{0} / \partial z$, they are calculated from Eq. (29), so that Eq. (20) yields strains $S_{4}$ and $S_{6}$ as follows :

$$
\begin{aligned}
S_{4} & =\tau\left(x+\Xi^{\prime \prime} \frac{\partial \phi^{\prime}}{\partial z^{\prime}}\right) \\
& -\frac{Q}{c_{22}^{\prime} I_{z}}\left\{\Xi^{\prime \prime} \frac{\partial \chi^{\prime}}{\partial z^{\prime}}+\frac{1}{2} \frac{c_{22}^{\prime}}{\widehat{c}_{44}}\left(1+\frac{2}{\Xi}\right) x z\right\}
\end{aligned}
$$

and

$$
\begin{aligned}
S_{6} & =\tau\left(-z+\Xi^{\prime} \frac{\partial \phi^{\prime}}{\partial x^{\prime}}\right)-\frac{Q}{c_{22}^{\prime} I_{z}} \\
& \times\left[\Xi^{\prime} \frac{\partial \chi^{\prime}}{\partial x^{\prime}}+\frac{1}{4} \frac{c_{22}^{\prime}}{\widehat{c}_{44}}\left\{\frac{\widehat{c}_{44}}{c_{66}^{\prime}}\left(1-\frac{2}{\Xi}\right) x^{2}\right.\right. \\
& \left.\left.+\left(1+\frac{2}{\Xi}-4 \frac{\widehat{c}_{44}}{c_{22}^{\prime}} \sigma_{z}\right) z^{2}\right\}\right]
\end{aligned}
$$

where,

$$
\Xi^{\prime \prime}=\sqrt{\frac{2 c_{66}^{\prime}}{\widehat{c}_{44}+c_{66}^{\prime}}}
$$

Consequently, stress $T_{6}$ is given as

$$
\begin{aligned}
T_{6} & =c_{66}^{\prime} \tau\left(-z+\Xi^{\prime \prime \prime} \frac{\partial \phi^{\prime}}{\partial x}\right)-\frac{c_{66}^{\prime} Q}{c_{22}^{\prime} I_{z}} \\
& \times\left[\Xi^{\prime \prime \prime} \frac{\partial \chi^{\prime}}{\partial x}+\frac{1}{4}\left\{\frac{c_{22}^{\prime}}{c_{66}^{\prime}}\left(1-\frac{2}{\Xi}\right) x^{2}\right.\right. \\
& \left.\left.+\frac{c_{22}^{\prime}}{\widehat{c}_{44}}\left(1+\frac{2}{\Xi}-4 \frac{\widehat{c}_{44}}{c_{22}^{\prime}} \sigma_{z}\right) z^{2}\right\}\right]
\end{aligned}
$$

where,

$$
\Xi^{\prime \prime \prime}=\Xi^{\prime} \Xi^{\prime \prime}=\frac{2 \sqrt{\hat{c}_{44}+c_{66}^{\prime}}}{\hat{c}_{44}+c_{66}^{\prime}}
$$

\section{4 Residual displacement $v_{y}$}

As $\partial S_{5} / \partial y=0$ is taken into account, strains $S_{2}, S_{4}$ and $S_{6}$ have the relation

$$
2 \frac{\partial^{2} S_{2}}{\partial z \partial x}=\frac{\partial}{\partial y}\left(\frac{\partial S_{4}}{\partial x}+\frac{\partial S_{6}}{\partial z}\right)
$$

Deriving $\partial S_{4} / \partial x$ and $\partial S_{6} / \partial z$ from Eqs. (34) and (35), substitution of the derivatives into Eq. (37) gives

$$
\begin{aligned}
& 2 \frac{\partial^{2} S_{2}}{\partial z \partial x}=\frac{\partial}{\partial y}\left[2 \tau \Xi^{\prime \prime \prime} \frac{\partial^{2} \phi^{\prime}}{\partial x \partial z}-\frac{Q}{c_{22}^{\prime} I_{z}}\right. \\
& \left.\quad \times\left\{2 \Xi^{\prime \prime \prime} \frac{\partial^{2} \chi^{\prime}}{\partial x \partial z}+\frac{c_{22}^{\prime}}{\widehat{c}_{44}}\left(1+\frac{2}{\Xi}-2 \frac{\bar{c}_{44}}{c_{22}^{\prime}} \sigma_{z}\right) z\right\}\right]
\end{aligned}
$$

Integration of Eq. (38) over the cross section and the relation of $S_{2}=\partial u_{y} / \partial y$ yield the displacement $u_{y}$ as

$$
\begin{aligned}
u_{y} & =x f_{z}(y)-\frac{Q}{c_{22}^{\prime} I_{z}}\left\{\Xi^{\prime \prime \prime} \chi^{\prime}+\frac{1}{4} \frac{c_{22}^{\prime}}{\hat{c}_{44}}\right. \\
& \left.\times\left(1+\frac{2}{\Xi}-2 \frac{\bar{c}_{44}}{c_{22}^{\prime}} \sigma_{z}\right) x z^{2}\right\}
\end{aligned}
$$

where the function $f_{z}(y)$ is a polynomial whose exact form is dependent upon the end conditions of the beam and which has a form of $\alpha y+\beta$ ( $\alpha, \beta$ : constant) to satisfy $\partial S_{4} / \partial y=0$. The derivative of displacement $u_{y}$ makes it possible to calculate $V(y)$ and $\Phi(y)$ from the relation of Eqs. (5) and (6). From the second relation of Eq. 7 ), the residual displacement $v_{y}$ is transformed into

$$
\begin{aligned}
v_{y} & =u_{y}-V(y)-x \Phi(y) \\
& =x f_{z}(y)-\frac{Q}{c_{22}^{\prime} I_{z}}\left\{\Xi^{\prime \prime \prime} \chi^{\prime}+\frac{1}{4} \frac{c_{22}^{\prime}}{\widehat{c}_{44}}\right. \\
& \left.\times\left(1+\frac{2}{\Xi}-2 \frac{\widehat{c}_{44}}{c_{22}^{\prime}} \sigma_{z}\right) x z^{2}\right\}-\frac{1}{A} \int_{-z_{0}}^{z_{0}} \int_{-x_{0}}^{x_{0}} u_{y} d x d z \\
& -\frac{x}{I_{z}} \int_{-z_{0}}^{z_{0}} \int_{-x_{0}}^{x_{0}} x u_{y} d x d z
\end{aligned}
$$

In Eq. (36) we neglect the torsional term, namely

$$
\tau\left(-z+\Xi^{\prime \prime \prime} \frac{\partial \phi^{\prime}}{\partial x}\right)=0
$$

Substitution of the $T_{6}$ and $\partial v_{y} / \partial x$ into the denominator of the right side in Eq. (13) gives

$$
\begin{aligned}
& \int_{-z_{0}}^{z_{0}} \int_{-x_{0}}^{x_{0}}\left(T_{6}-c_{66}^{\prime} \frac{\partial v_{y}}{\partial x}\right) d x d z=\frac{Q}{K} \\
& =\frac{c_{66}^{\prime}}{c_{22}^{\prime}} \frac{Q}{I_{z}}\left[\sigma_{z} I_{x}-\frac{1}{4}\left\{\frac{c_{22}^{\prime}}{\widehat{c}_{44}}\left(1+\frac{2}{\Xi}\right) I_{x}\right.\right. \\
& \left.\left.+\frac{c_{22}^{\prime}}{c_{66}^{\prime}}\left(1-\frac{2}{\Xi}\right) I_{z}\right\}-\frac{A}{I_{z}} \int_{-z_{0}}^{z_{0}} \int_{-x_{0}}^{x_{0}} \Xi^{\prime \prime \prime} x \chi^{\prime} d x d z\right]
\end{aligned}
$$

where,

$$
I_{x}=\int_{-z_{0}}^{z_{0}} \int_{-x_{0}}^{x_{0}} z^{2} d x d z
$$

Thus, we find that the denominator of the right side in Eq. (13) is given as a function of the harmonic function $\chi^{\prime}$.

\section{Derivative of harmonic function $\chi^{\prime}$}

The equations of the boundaries are two of $x^{\prime}=$ $\pm x_{0}^{\prime}\left(x_{0}^{\prime}=x_{0} / \Xi^{\prime \prime}\right)$ and $z^{\prime}= \pm z_{0}^{\prime}\left(z_{0}^{\prime}=z_{0} / \Xi^{\prime}\right)$. From Eq.

(33) the boundary condition at $x^{\prime}= \pm x_{0}^{\prime}$ is

$$
\begin{aligned}
& \frac{\partial \chi^{\prime}}{\partial x^{\prime}}=\frac{1}{2}\left(1-\frac{1}{2} \Xi\right) x_{0}^{\prime 2}-\frac{1}{2}\left(1+\frac{1}{2} \Xi-2 \Xi^{\prime} \sigma_{z}\right) z^{\prime 2} \\
& \quad\left(-z_{0}^{\prime}\left\langle z^{\prime}\left\langle z_{0}^{\prime}\right\rangle\right)\right.
\end{aligned}
$$

while the boundary condition at $z^{\prime}= \pm z_{0}^{\prime}$ is

$$
\begin{aligned}
& \frac{\partial \chi^{\prime}}{\partial z^{\prime}}=\left\{\mp\left(1+\frac{1}{2} \Xi\right) z_{0}^{\prime}-\frac{c_{42}^{\prime}}{\widehat{c}_{44}}\left(2 y_{0}-y\right)\right\} x^{\prime} \\
& \quad\left(-x_{0}^{\prime}<x^{\prime}<x_{0}^{\prime}\right)
\end{aligned}
$$

We introduce a new function $\bar{\chi}$ by the equation 


$$
\begin{aligned}
\bar{\chi} & =\chi^{\prime}-\frac{1}{6}\left[\left(1+\frac{1}{2} \Xi\right) x^{\prime 3}\right. \\
& \left.-3\left\{\left(1+\frac{1}{2} \Xi\right) x^{\prime} z^{\prime 2}+\frac{2 c_{42}^{\prime}}{\hat{c}_{44}}\left(2 y_{0}-y\right) x^{\prime} z^{\prime}\right\}\right]
\end{aligned}
$$

Then $\bar{\chi}$ is a plane harmonic function within the rectangle, $\partial \bar{\chi} / \partial z^{\prime}$ vanishes at $z^{\prime}= \pm z_{0}^{\prime}$, and the condition at $x^{\prime}$ $= \pm x_{0}^{\prime}$ becomes

$$
\frac{\partial \bar{\chi}}{\partial x^{\prime}}=-\frac{1}{2} \Xi x_{0}^{\prime 2}+\Xi^{\prime} \sigma_{z} z^{\prime 2}+\frac{c_{42}^{\prime}}{\bar{c}_{44}}\left(2 y_{0}-y\right) z^{\prime}
$$

Now when $-z_{0}^{\prime}\left\langle z^{\prime}<z_{0}^{\prime}\right.$, the function $z^{2}$ can be expanded in a Fourier's series ${ }^{(5)}$ as follows :

$$
z^{\prime 2}=\frac{z_{0}^{\prime 2}}{3}+\frac{4 z_{0}^{\prime 2}}{\pi^{2}} \sum_{n=1}^{\infty} \frac{(-1)^{n}}{n^{2}} \cos \frac{n \pi z^{\prime}}{z_{0}^{\prime}}
$$

Hence, $\bar{\chi}$ can be expressed in the form

$$
\begin{aligned}
\bar{\chi} & =\left(-\frac{1}{2} \Xi x_{0}^{\prime 2}+\frac{1}{3} \Xi^{\prime} \sigma_{z} z_{0}^{2}\right) x^{\prime} \\
& +\frac{c_{42}^{\prime}}{\bar{c}_{44}}\left(2 y_{0}-y\right) x^{\prime} z^{\prime}+\Xi^{\prime} \sigma_{z} \frac{4 z_{0}^{\prime 3}}{\pi^{3}} \\
& \times \sum_{n=1}^{\infty} \frac{(-1)^{n}}{n^{3}} \frac{\sinh \frac{n \pi x^{\prime}}{z_{0}^{\prime}}}{\cosh \frac{n \pi x_{0}^{\prime}}{z_{0}^{\prime}}} \cos \frac{n \pi z^{\prime}}{z_{0}^{\prime}}
\end{aligned}
$$

and, by means of this relation and Eq. (45), $\chi^{\prime}$ can be written down, so that the harmonic function $\chi^{\prime}$ which includes the torsional term becomes

$$
\begin{aligned}
\chi^{\prime} & =\frac{1}{\Xi^{\prime \prime \prime}}\left\{\left(-\frac{1}{2} \frac{c_{22}^{\prime}}{c_{66}^{\prime}} x_{0}^{2}+\frac{1}{3} \sigma_{z} z_{0}^{2}\right) x\right. \\
& +\frac{1}{6}\left(\frac{1}{2} \frac{c_{22}^{\prime}}{c_{66}^{\prime}}+\frac{\widehat{c}_{44}^{\prime}}{c_{66}^{\prime}} \frac{1}{\Xi^{\prime}}\right) x^{3} \\
& \left.-\frac{1}{2}\left(\frac{1}{2} \frac{c_{22}^{\prime}}{\widehat{c}_{44}}+\frac{1}{\Xi^{\prime}}\right) x z^{2}\right\}+\frac{\sigma_{z}}{\Xi^{\prime 2}} \frac{4 z_{0}^{3}}{\pi^{3}} \\
& \times \sum_{n=1}^{\infty} \frac{(-1)^{n}}{n^{3}} \frac{\sinh \left(n \pi \sqrt{\frac{\widehat{c}_{44}}{c_{66}^{\prime}}} \frac{x}{z_{0}}\right)}{\cosh \left(n \pi \sqrt{\frac{\bar{c}_{44}}{c_{66}^{\prime}}} \frac{x_{0}}{z_{0}}\right)} \cos \frac{n \pi z}{z_{0}}
\end{aligned}
$$

The derivative of the harmonic function $\chi^{\prime}$ makes it possible to calculate the shear coefficient $K$.

\section{Calculation of shear coefficient $K$ and its calculated values}

From the relation of Eq. (42) the shear coefficient $K$ is given as

$$
\begin{aligned}
& K=\frac{c_{22}^{\prime} I_{z}}{c_{66}^{\prime}[}\left[\sigma_{z} I_{x}-\frac{1}{4}\left\{\frac{c_{22}^{\prime}}{\bar{c}_{44}}\left(1+\frac{2}{\Xi}\right) I_{x}+\frac{c_{22}^{\prime}}{c_{66}^{\prime}}\left(1-\frac{2}{\Xi}\right) I_{z}\right\}\right. \\
&\left.-\frac{A}{I_{z}} \int_{-z_{0}}^{z_{0}} \int_{-x_{0}}^{x_{0}} \Xi^{\prime \prime \prime} x \chi^{\prime} d x d z\right]
\end{aligned}
$$

As a result of calculation, we find that the shear coefficient $K$ in Timoshenko's beam theory for trigonal crystals of rectangular cross section, derived using Cowper's definition is

$$
K=\frac{10 c_{22}^{\prime}}{11 c_{22}^{\prime}+\sqrt{2 \hat{c}_{44}\left(\widehat{c}_{44}+c_{66}^{\prime}\right)}}
$$

As is apparent from Eq. (51), $K$ of the rectangular cross section is independent of a thickness-to- ${ }^{-}$idth ratio. In case of an isotropic material, when Poisson's ratio is taken as $\sigma$, the constants $c_{22}^{\prime}, \hat{c}_{44}$ and $c_{66}^{\prime}$ have the relation

$$
\bar{c}_{44}=c_{66}^{\prime}=\frac{c_{22}^{\prime}}{2(1+\sigma)}
$$

Substituting this into Eq. (51), the shear coefficient $K$ for the isotropic material becomes

$$
K=\frac{10(1+\sigma)}{12+11 \sigma}
$$

This result agrees completly with that obtained by $\mathrm{G}$. R. Cowper ${ }^{(2)}$. The following shows an example of the calculated results. In the calculation the constants of Refs. ${ }^{(6),(7)}$ are used.

Figure 2 shows the relationship of the shear coefficient $K$ and a cut angle $\theta$ rotated about the $x^{-}$ axis. $K$ varies with the cut angle $\theta$ and has a value of $0.796-0.867$ when the cut angle $\theta$ varies from $-90^{\circ}$ to $+90^{\circ}$.

Figure 3 shows the relationship of $K$ and $\theta$ for lithium tantalate rotated about the $x$-axis. $K$ has a value of $0.834-0.857$ when the cut angle $\theta$ varies from $-90^{\circ}$ to $+90^{\circ}$.

Figure 4 shows the relationship of $K$ and $\theta$ for lithium niobate rotated about the $x$-axis. $K$ has a

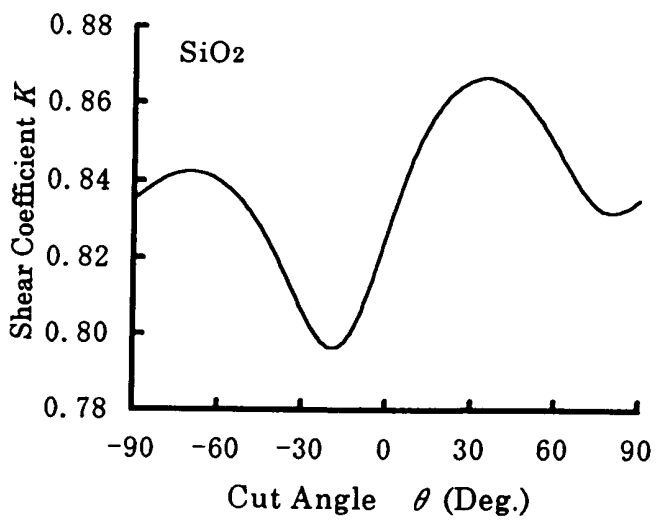

Fig. 2 Relationship of the shear coefficient $K$ and a cut angle $\theta$ rotated about the $x$-axis

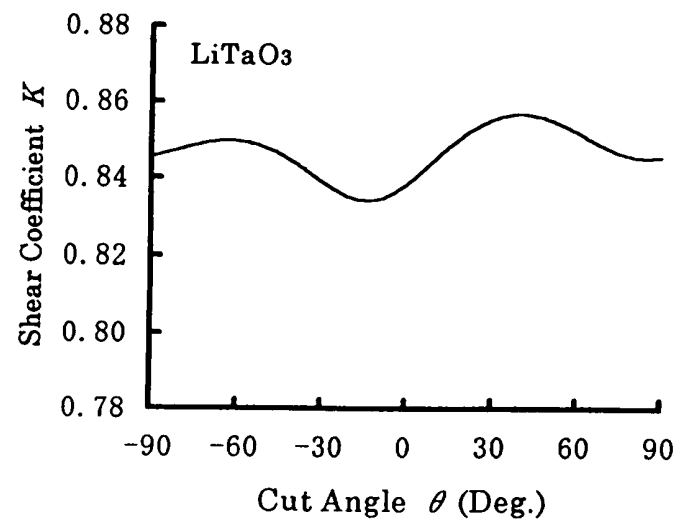

Fig. 3 Relationship of the shear coefficient $K$ and a cut angle $\theta$ for lithium tantalate rotated about the $x$ axis 
Table 1 Comparison of our values of $K$ for quartz crystal, lithium tantalate, and lithium niobate, and those $^{(2)}$ for isotropic material obtained by other authors

\begin{tabular}{l|c} 
& $K$ \\
\hline Timoshenko & 0.667 \\
Mindlin & 0.822 \\
Goodman $(\sigma=1 / 3)$ & 0.870 \\
Roark & 0.833 \\
Cowper $(\sigma=0)$ & 0.833 \\
$\quad(\sigma=0.3)$ & 0.850 \\
$\quad(\sigma=1 / 2)$ & 0.870 \\
\hline The author & $0.796 \sim 0.867$ for Q.C. \\
& $0.834 \sim 0.857$ for L.T. \\
& $0.831 \sim 0.863$ for L.N
\end{tabular}

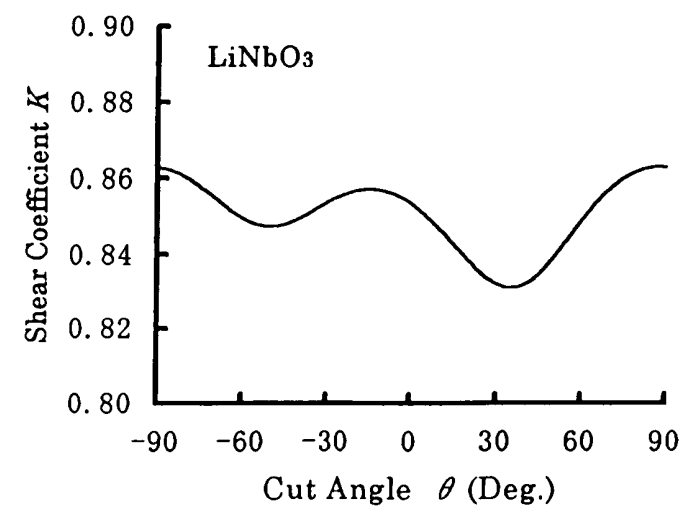

Fig. 4 Relationship of the shear coefficient $K$ and a cut angle $\theta$ for lithium niobate rotated about the $x$ axis

value of $0.831-0.863$ versus a variation of the abovementioned cut angle.

Table 1 compares our values of $K$ for quartz crystal (Q. C.), lithium tantalate (L. T.), and lithium niobate (L. N.) with those for the isotropic material obtained by other authors. With the exception of Timoshenko's value, the foregoing results almost include those obtained by other authors and agree fairly well with each other.

\section{Conclusions}

In this paper, the shear coefficient $K$ in Timoshenko's beam theory for trigonal crystals of the rectangular cross section that are anisotropic has been derived using Cowper's definition. It was shown that the shear coefficient $K$ is given as a function of the transformed elastic stiffness constants $c_{22}^{\prime}, \hat{c}_{44}, c_{66}^{\prime}$ only and is independent of a thickness-to-width ratio of the cross section. In addition, $K$ for quartz crystal, lithium tantalate, and lithium niobate versus a cut angle $\theta$ was calculated, so that it was found to be very dependent upon the cut angle $\theta$.

For the coming subject, this $K$ will be applied to a vibration analysis of flexural mode trigonal crystal resonators, and the influence by $K$ will be clarified on resonance frequency and frequency temperature behavior for the flexural mode resonators.

\section{Acknowledgment}

The author expresses his thanks to M. Kato for his help in the numerical calculation by computer.

\section{References}

(1) Timoshenko, S. P., Vibration Problems in Engineering, (1937), D. Van Nostrand Co. Inc., New York.

(2) Cowper, G. R., The Shear Coefficient in Timoshenko's Beam Theory, Journal of Applied Mechanics, Vol. 33 (1966), p. 335.

( 3 ) Mindlin, R. D. and Deresiewicz, H., Timoshenko's Shear Coefficient for Flexural Vibrations of Beams, Technical Report No. 10, ONR Project NR 064-388, (1953), Department of Civil Engineering, Columbia University, New York, N. Y.

(4) Goodman, L. E., discussion of paper, Flexural Vibrations in Uniform Beams According to the Timoshenko Theory, by R. A. Anderson, Journal of Applied Mechanics, Vol. 21 (1954), p. 202.

(5) Love, A. E. H., A treatise on the Mathematical Theory of Elasticity, fourth edition, (1952), Cambridge University Press, Cambridge, England.

(6) Koga, I., Aruga, M. and Yoshinaka, Y., Theory of Plane Elastic Waves in a Piezoelectric Crystalline Medium and Determination of the Elastic and Piezoelectric Constants of Quartz, Phys. Rev. Vol. 109 (1958), p. 1467.

( 7 ) Smith, R. T. and Welsh, F. S., Temperature Dependence of the Elastic, Piezoelectric, and Dielectric Constants of Lithium Tantalate and Lithium Niobate, Journal of Applied Physics, Vol. 42 (1971), p. 2219. 